\title{
Los procesos de subjetividad y participación política de estudiantes de psicología de Bogotá
}

\section{Subjectivity and political participation processes of Psychology students of Bogotá}

\author{
Martha Cecilia Lozano Ardila* \\ Universidad Católica de Colombia, \\ Bogotá, Colombia
}

Recibido: 23 de abril de 2008

Revisado: 14 de junio de 2008

Aceptado: 26 de junio de 2008

\section{Resumen}

A pesar de ser la política un tema de interés para las Ciencias Humanas y Sociales, son pocos los estudios que se han llevado a cabo en Colombia sobre el tema de la subjetividad política de los jóvenes. Por lo anterior esta investigación tuvo como objetivo interpretar la subjetividad y participación política de los estudiantes universitarios en Bogotá. Se trabajó con base en el enfoque de la investigación cualitativa mediante la entrevista y el grupo focal, por consiguiente, se trabajó con 20 estudiantes, entre los 18 y 25 años, de estrato socioeconómico medio, hombres y mujeres. El enfoque de la investigación fue de carácter histórico-hermenéutico a través del método narrativo, desde la perspectiva de Bruner (2002). Se concluyó que los procesos de subjetividad política de los jóvenes participantes en el estudio están mediados por la forma en que conciben la política y la participación en ésta y la influencia de agentes de socialización como la familia, los pares e incluso el Estado.

Palabras clave: juventud, subjetividad política, participación política, narrativa.

* Correspondencia: Martha Lozano. Facultad de Psicología. Universidad Católica de Colombia. Correo electrónico: marthalo2001@yahoo.com. Dirección postal: Calle 47 No. 13 -32, Bogotá, Colombia. 


\section{Abstract}

Despite being an issue of policy concern for the human and social sciences, few studies have been carried out in Colombia on the theme of subjectivity youth policy. That is why this research aimed to interpret the subjectivity and political participation of university students from the city of Bogota. It worked within the focus of qualitative research through interviews and focus group. We worked with 20 students, between 18 and 25 years of socioeconomic environment, men and women. The focus of the research was of historic nature - hermeneutical method through the narrative from the perspective of Bruner (2002). It was concluded in the investigation that the political processes of subjectivity of the young participants in the study are mediated by the way they conceive politics and participation in and influence of socialization agents such as family, peers and even the state.

Key words: youth, political subjectivity, political participation, narrative.

\section{Introducción}

Si se parte de la historia del desarrollo de la juventud y su participación en los procesos políticos y sociales, se hace necesario identificar las nuevas condiciones que encierra la sociedad contemporánea en relación con los diversos procesos de participación en lo político y en lo social (Alba, 1975; Levi \& Schmitt, 1996; Martín, 1998; Cohen, 1999), procesos que difieren, muchas veces, por asuntos de edad, sexo o clase social.

Actualmente, la participación política de los jóvenes ha adquirido un nuevo sentido, debido a los giros que implican las tensiones de integración de los jóvenes al desarrollo social, económico y cultural, propiciadas por los procesos de globalización y del neoliberalismo (Held, 1997; de Sousa, 1998; Lechner, 1995; 1999). Si bien es cierto que en las tendencias actuales de la democracia se han abierto espacios de participación a todos los ciudadanos, aún se carece de coherencia entre lo que en la democracia se expresa y lo que las personas, en particular los jóvenes, realizan como sujetos políticos (Rauber, 2006).

Por esto disciplinas como la Psicología Política, a través del estudio de los procesos objetivos y subjetivos de socialización política, aporta a los análisis sobre los procesos de expresión de la subjetividad política mediante las prácticas de participación, las creencias, los sentidos y sin sentidos sobre la política (Brown, 1999; Brewer, 2001).
Es claro que en las últimas décadas en las sociedades se han dado cambios democráticos relevantes en los que se hacen visibles los y las ciudadanas a través de los mecanismos de participación; sin embargo, aún existen circunstancias que distancian a los jóvenes y a muchos adultos de la esfera de la política. Desde su subjetividad los jóvenes han generado formas diferentes de expresión de lo político a través del arte (Huntington, 1997; Pérez, 2000), de la participación formal en instancias de la política, como los comités comunitarios o los consejos de juventud o mediante el rechazo de la política por las problemáticas que la circundan, a saber: la corrupción, el tradicionalismo o las hegemonías por tradición de partido o de familia (Calcagno \& Calcagno, 1999; Arendt, 2001).

Hoy en día hay formas "no convencionales" de expresar su rechazo a prácticas políticas que afectan su posibilidad de inclusión a la fuerza laboral, el reconocimiento de la diferencia, de los Derechos Humanos o de la protección del medio ambiente. Los jóvenes integran una visión diferente de la política y aportan, desde su perspectiva como jóvenes, a la conformación y resignificación de la política y la participación en ésta (Pastor, 2002).

De igual forma, en la actualidad, las prácticas políticas de grupos diferentes de jóvenes no se adhieren ni al antiguo modelo de la política, ni tampoco a las nuevas prácticas políticas, que no terminan de imponerse. Los grupos de jóvenes cuya acción tiene un sentido político o sociopolítico desarrollan lógicas de acción distintas a las 
de la política convencional; promueven modos de agenciamiento enmarcadas en sus subjetividades enraizadas profundamente en la vida cotidiana (Merlino \& Roqué, 2004; Ferrándiz \& Feixa, 2005). No hay duda de que estas formas de expresión de los jóvenes, como sujetos políticos, aportan en mayor medida al proceso de mutación de la política, a pesar de la tendencia tradicionalista de los políticos (Maffesoli, 2004).

Las trayectorias políticas de la primera mitad del siglo XX se constituyeron en soporte para que la participación de los jóvenes pasara de una participación política crítica, movimientos de resistencia y luchas a un estado de poco interés por la política convencional, así como a una dispersión cada vez mayor frente a la política y a las formas de expresión de algunos grupos de jóvenes fuera de la tradición política o del retraimiento de otros, todo esto ante la crisis de la política (Balardini, 2000).

En consecuencia, la participación juvenil en lo político, en cuanto a la toma de decisiones, es considerada relativamente baja. Por otra parte, la profesionalización de la política, por medio de sus diferentes ramas de aplicación (Derecho, Psicología Política, Filosofía Política), ha permitido resaltar y nutrir el trabajo que la política ha hecho desde prácticas sociales y de cobertura fragmentada (Arendt, 2001). De alguna manera, la política ha perdido su norte para fundirse en lo económico y en algunas intervenciones de carácter social.

Es aquí en donde la Psicología, como ciencia que estudia el comportamiento y las interacciones del hombre con su medio, ve la necesidad y, sobre todo, la importancia de estudiar no sólo desde el fenómeno de la conducta visible, sino también desde la subjetividad, el proceso general y complejo de la configuración política del ser humano a través de los procesos de socialización, con la intención de contribuir en la comprensión del ser humano como sujeto (Sabucedo, 1996; D’Adamo, García \& Montero, 1995).

De ahí la importancia de recurrir al método narrativo como estrategia de recuperación de la subjetividad y la participación política de los jóvenes participantes en el estudio (Bruner, 2002). Se tuvo en cuenta que para el análisis de las narrativas, la condición principal para llegar a la comprensión de las subjetividades de los jóvenes frente a la política y su participación en ella, es la interacción comunicativa entre los jóvenes y el investigador, es una condición necesaria para alcanzar una adecuada comprensión de las creencias, significados, de las expectativas y las decisiones políticas de los participantes.

Para el análisis de las narrativas se incluyeron como ejes de reflexión estamentos sociales vinculados con la política nacional y con los actores que participan en política, tales como los valores políticos, ideologías políticas, instituciones políticas, organizaciones políticas, grupos políticos, las significación de las relaciones políticas, los significados sobre los procesos políticos del país, el sentido de sus acciones y el sentido otorgado a los discursos políticos.

En el contexto global se hace evidente la manifestación de la subjetividad política en acciones de organización, participación o expresión política de los jóvenes, a través de sus propias formas de ser en el mundo, diferentes a las formas tradicionales de la política. En todos los niveles del discurso y formas de acción política se encuentran las huellas de procesos de socialización, tanto objetiva como subjetiva diferentes, en las cuales características como el género, la clase, etnicidad, edad, origen, y posición, u otras formas de pertenencia grupal de los actores políticos juegan un rol fundamental en las formas de pensar y participar en la política. Es necesario tener en cuenta que las condiciones históricas, sociales, culturales y políticas no son estáticas; por lo tanto, para entender la construcción del concepto de subjetividad política en los jóvenes es necesario ubicarse en un marco histórico y social que depende de la construcción cultural de cada sociedad, ya que, según las diferentes épocas, cambia su sentido y dinámica de funcionamiento (Garretón, 2000; Huergo, 2004).

En el contexto colombiano se han realizado pocas investigaciones sobre este tema, y tal como 
lo manifiestan Benedicto y Morán (2003) es importante llevar a cabo estudios que permitan comprender por qué aumenta el número de jóvenes políticamente desinformados, desconfiados de la política y de los políticos. En este sentido, destacan la necesidad de investigaciones acerca del estado actual de los conocimientos políticos y sociales, creencias, actitudes y valores de los jóvenes; también se añade que existen pocos países en los que las respuestas a estas preguntas están disponibles, pero su valor se multiplicaría si existiera algún tipo de coordinación orientada a facilitar las comparaciones internacionales.

Para los propósitos de esta investigación se definió la política como ciencia y arte. Como ciencia, proporciona una serie de conocimientos relativos al Estado y como arte, investiga las soluciones de los problemas concretos que constituyen los fines del Estado. Su significado es polisémico, aunque siempre se concuerda que pertenece a las relaciones entre los hombres y mujeres, en la que hay una compleja interacción que muestra una diferenciación jerárquica entre gobernantes y gobernados, en nombre de una finalidad social que se manifiesta en relaciones de representación y de participación, de un poder político (Sartori, 1995).

En sentido amplio, la política se manifiesta como el estudio de la organización y gobierno de las comunidades humanas, y su dominio se extiende al conocimiento de todas las formas de gobierno que han existido y que existen, a las organizaciones sociales que ha tenido y tiene la sociedad humana, al desenvolvimiento gradual de las instituciones y al examen del pensamiento y las doctrinas, hechos y acontecimientos, dirigidos o vinculados con las relaciones de poder entre los hombres de una comunidad en sí misma (Sartori, 1995; Sabine, 2002).

Por lo tanto, incluye todas aquellas actividades formales e informales que conforman las instituciones de gobierno de un Estado. Las actividades formales están dadas por la Constitución o por la costumbre y la tradición de la vida política de un país; son todas aquellas instituciones reconocidas por todos como las que determinan el modo de ejercer autoridad y gobierno sobre un territorio o nación. Le corresponde a los mecanismos para generar los poderes del Estado y su funcionamiento. Ejemplos de esto son las elecciones o el proceso de tramitación de un proyecto de ley. Las actividades informales están dadas por el modo de ejercer el poder en un gobierno o en la vida pública de una nación; estos modos están validados por su uso o legitimados por la opinión pública.

De este modo, la vida política está estrechamente ligada al gobierno y a la vida pública. Al gobierno, pues considera su gestación y continuidad, las relaciones internacionales entre los estados y la mejor forma de gobernar un país; a la vida pública, pues todos estos hechos son de relevancia para toda la sociedad, si se piensa en un estilo de vida y en un régimen social democrático.

En pocas palabras, se puede decir que política es el conjunto de prácticas de organización y gobierno de la vida en sociedad. Gobernar un territorio significa mucho más que el mero hecho de prestar a sus habitantes una serie de servicios; esto significa la posibilidad de desarrollar nuevas formas de regulación social, de fabricar y mantener los lazos sociales sin los cuales vivir en comunidad deja de tener sentido. También significa favorecer la apropiación de la política por parte de los ciudadanos, de los diversos actores, al dar sentido a la acción colectiva, al concebir y desarrollar proyectos comunes, en una visión de conjunto percibida por todos como legítima (Benedicto \& Moran, 2003).

La política incluye aspectos como la participación, entendida como el conjunto de actividades, interacciones, comportamientos, acciones y actitudes que se dan en una sociedad de forma individual o colectiva por los ciudadanos, grupos, partidos e instituciones, las cuales van dirigidas a explicar, demandar, influir o tomar parte en el proceso de decisiones políticas, en el reparto autoritario de valores.

Asimismo, la participación política es entendida desde las actividades voluntarias mediante las cuales los miembros de una sociedad participan en la elección de sus gobernantes y, directa o indirectamente, en la elaboración de la política gu- 
bernamental. La participación política no se debe restringir a unas determinadas acciones, sean individuales o colectivas, sino que en una dimensión más amplia, ésta contempla una serie de comportamientos convencionales que son propiciadas, o impulsadas desde las instancias del poder constituido (Barcena, 1997).

En la participación política convencional se encuentra por ejemplo votar, tomar parte en campañas, actividades comunitarias y buscar ayuda oficial para resolver problemas comunitarios (Sabucedo, 1996). La participación política no convencional hace referencia a la heterogeneidad de actividades que recogen actuaciones legales como: manifestaciones, peticiones, marchas, los paros cívicos, los bloqueos de vías públicas, la desobediencia civil y todas las que se expresan en los distintos grados de violencia y que suponen un enfrentamiento con la legalidad establecida. Además de éstas, en la actualidad se encuentran formas de expresión a través del arte, la música - la resistencia a las políticas, producto de la neoliberalización (Nateras, 2002; Pereira \& Villadiego, 2006).

Existen otras formas de participación a través de lo institucional, que se define como la acción de participar en un campo o actividad. Es la forma en que el individuo se involucra en la dialéctica de la sociedad buscando el espacio para generar un efecto en ella. Ahora bien, participar, en sentido estricto, es desarrollar una acción colectiva $y$, por lo tanto, organizada, en interés de terceros, orientada al bien común y a necesidades sociales. Cuando se cumplen estas condiciones se habla de participación social; en este sentido, la pertenencia a una asociación, en determinadas condiciones; el voluntariado organizado; la pertenencia a un movimiento social, a un partido político, a un sindicato; ser delegado(a) de un aula o representante en el consejo escolar son formas de participación social.

En un sentido amplio se pueden entender como formas de participación: el voto, el trabajo, el estudio, participar puntualmente en una campaña, en una manifestación. Todas estas actividades tienen un carácter individual o colectivo, independientemente de que sean el resultado de una iniciativa personal o que sean propuestas por una asociación o un movimiento social. Son formas de participación, en la medida en que contribuyen al bien común. Con frecuencia es posible participar a nivel individual y puntualmente sólo porque existen organizaciones, que de manera estable y formalizada, impulsan y ofrecen un marco para el desarrollo de estas acciones. (Benedicto \& Morán, 2003).

En cuanto a la actitud política, ésta hace referencia al conjunto de creencias y valores que orientan el pensamiento de una persona con respecto a un tema. La actitud política se constituye a partir de predisposiciones adquiridas por los ciudadanos, las cuales se manifiestan a través de distintas formas de participación política. Según Sabucedo (1996) y D’Adamo, García y Montero (1995), las actitudes corresponden a una organización relativamente duradera, que interrelacionadas describen, evalúan y abogan por un objeto o situación y en ambos casos son importantes en el grado de participación política. El análisis hecho a partir de las creencias y actitudes tiene una mayor relevancia, porque a través de ellas se conjugan los factores subjetivos individuales con las condiciones sociales, en las que se encuentran las motivaciones más prometedoras de la participación política.

Finalmente, se incluye el concepto de democracia, que se refiere al sistema político por el que el pueblo de un Estado ejerce su soberanía mediante cualquier forma de gobierno que haya decidido establecer. En la democracia, la autoridad suprema la ejercen, en su mayor parte, los representantes elegidos por sufragio popular en reconocimiento de la soberanía nacional. Dichos representantes pueden ser sustituidos por el electorado, de acuerdo con los procedimientos legales de destitución y referendo y son, al menos en principio, responsables de su gestión de los asuntos públicos ante el electorado. En el sistema democrático colombiano, el pueblo elige tanto al jefe del poder Ejecutivo como al cuerpo responsable del Legislativo. La esencia del sistema democrático supone la participación de la población en el nombramiento de representantes para 
el ejercicio de los poderes ejecutivo y legislativo del Estado, independientemente de que éste se rija por un régimen monárquico o republicano.

La concepción de Rousseau con respecto a la democracia, plantea al individuo como centro del Estado, en el que la voluntad general debe dirigir las fuerzas del Estado, puesto que la voluntad de algunos da como resultado un mal gobierno, debido a que, según el autor, el poder soberano tiene sus límites en el poder popular (Rozo, 1990).

Las otras categorías de análisis relevante para los propósitos de esta investigación son la subjetividad política y la participación política. La subjetividad en sentido amplio se refiere al núcleo fundamental inherente a la condición humana como diferenciadora del ser humano de las demás sustancias conocibles y observables. A partir de la subjetividad cada ser humano constituye un suceso independiente en el universo, un sistema motivacional, cognitivo y afectivo singular, único, delimitado e integrado. Es el centro de la conciencia, el juicio y la vida emocional (Goolishian \& Anderson, 1998); asimismo, corresponde al proceso de socialización objetiva y subjetiva.

La socialización se entiende como el proceso mediante el cual un individuo aprende a ser miembro de una sociedad y a ser él mismo, asumiendo los roles y los estatus asignados o adquiridos en ese proceso, el cual le servirá para desempeñarse en la sociedad; esto implica variables psicológicas, sociales y ambientales que se dan progresivamente, de acuerdo con el proceso de desarrollo evolutivo (Sabucedo, 1996; Berger \& Luckman, 1999).

La subjetividad política se constituye en el "espacio" por excelencia para la producción de sentido, para este caso el político; es sobre éste que actúan los jóvenes, adultos e, incluso niños y niñas para construir realidades posibles o transformar las existentes. La subjetividad es emancipatoriamente potente (de Sousa, 1994; Guattari, 1998) e incluye el conocimiento, las construcciones simbólicas e imaginarias de los saberes que entretejen lo simbólico, lo social y lo singular para la construcción de la realidad.
Por consiguiente, la subjetividad política requiere de autoconciencia y un autoconocimiento (dado su carácter histórico y social), por los sujetos de su locus en la sociedad y la disposición a participar para cambiar o eliminar aquellas estructuras que influyen negativamente sobre las relaciones sociales que configuran las identidades, lo que constituye formas particulares de socialización política, en tanto formas de aprendizaje político, manifiesto o no, permanente, deliberado o no, que afecta el comportamiento, las actitudes y las características de la personalidad en lo político.

En el campo de la Psicología, la concepción de socialización política ha sido abordada en una primera etapa de su estudio como la explicación de los factores que determinan un tipo de conducta política manifiesta como la participación en elecciones, la identificación con grupos o partidos políticos, la filiación a ciertas opciones políticas. La noción proveniente de la psicología política, propone la existencia de un proceso de transmisión y de modificación que reproduce y modifica los aspectos directamente relacionados con el modo de organización y de participación del individuo en los asuntos públicos (Bonilla-Castro \& Rodríguez, 1997).

Según Restrepo, Ortiz, Parra, y Medina, (1998) la socialización política corresponde a todos aquellos procesos de enseñanza y aprendizaje (formales o no formales) en una cultura de la sociabilidad que habilitan como miembros (como integrantes, como súbditos o ciudadanos) de instituciones (familiares, vecinales o municipales, nacionales o transnacionales, o un conjunto de todas ellas), a los individuos que han habitado o habitan un Estado o país. El proceso de socialización es la forma en que se interioriza la realidad social. Previamente, para Mead (1982), el proceso de socialización está orientado en función de lo que las cosas significan para un individuo y éstas también se van dinamizando mediante procesos de interpretación subjetiva. Con base en estos referentes, se formuló la siguiente pregunta de investigación: ¿cuáles son los procesos de subjetividad y de participación política de estudiantes de Psicología en Bogotá, Colombia? 


\section{Objetivos}

\section{Objetivo general}

Identificar los procesos de subjetividad y participación política de estudiantes de Psicología en Bogotá, Colombia.

\section{Objetivos específicos}

- Establecer, por medio del análisis de narrativas, los procesos de subjetividad y participación política en jóvenes estudiantes de Psicología en Bogotá, Colombia.

- Identificar, por medio del análisis de narrativas, la comprensión del concepto de política que poseen los jóvenes participantes en el estudio.

- Comprender, desde el análisis de narrativas, los procesos de subjetividad y participación política en jóvenes estudiantes de Psicología en Bogotá, Colombia.

\section{Categorías}

- Subjetividad política,

- Participación política,

- Socialización política.

\section{Método}

Para llevar a cabo este estudio se optó por una postura epistemológica histórico-hermenéutica y, con base en ella, se empleó el método narrativo (Bruner, 1997; 2002). La hermenéutica se puede tomar como arte y como ciencia, es decir, arte y ciencia de interpretar textos. Los textos no sólo son los escritos, sino también los hablados, los actuados y aún de otros tipos (estéticos, visuales); éstos van más allá de la palabra y el enunciado. Una característica esencial del enfoque hermenéutico es la polisemia, es decir, el múltiple significado que posea el texto que se vaya a interpretar, el cual debe traspasar el sentido superficial para llegar a uno más profundo que esté vinculado al sentido auténtico que le dio el autor; esto se logra por medio de la interpretación, que comienza como hipótesis para transformarse en tesis (Beuchot, 2002).
El análisis narrativo se debe entender como un enfoque que adquiere consistencia en lo individual, lo colectivo, lo histórico, lo social y cultural a través de la comprensión de las trayectorias y las tramas de existencia de los participantes (Lieblich, Tuval-Mashlach \& Zilber, 1998).

\section{Participantes}

Se seleccionaron, de forma intencional para llevar a cabo el estudio, 20 estudiantes de Psicología, entre hombres y mujeres. El grupo corresponde a un nivel socioeconómico medio, con edades comprendidas entre los 18 y 25 años.

\section{Instrumentos}

Para el desarrollo de la investigación se tuvieron en cuenta la entrevista individual y el grupo focal, por ser considerados instrumentos válidos y confiables para la recolección de la información pertinente en la investigación. Para definir los criterios de selección que se utilizaron a la hora de seleccionar el grupo focal se tuvieron en cuenta las características demográficas, la edad y el sexo.

\section{Procedimiento}

Selección de participantes de acuerdo con los criterios de edad, nivel socioeconómico y disciplina de formación (Psicología). El diseño de los instrumentos se hizo al tomar como referencia las orientaciones teóricas previamente consultadas y los criterios de diseño propuestos en la investigación de tipo cualitativo (Valles, 1998; Denzin \& Lincoln, 2003).

Además, y como parte de los objetivos de investigación, el diseño del instrumento se hizo teniendo en cuenta el contexto para la realización del trabajo de campo y garantizar la credibilidad y la verosimilitud de los datos (Pourtois \& Desmet, 1992; Josselson \& Lieblich, 1993).

\section{Resultados}

Con respecto al concepto de política, se encontró que los participantes la conciben como una forma 
de gobernar, administrar y organizar la sociedad y los individuos que la conforman; según ellos, esta organización está dada a partir de una serie de normas y de leyes que permiten regular el comportamiento de los individuos en la sociedad.

A la vez, en la entrevista a grupo focal se evidenció que el concepto de política está relacionado con la legitimación del poder representado en los políticos y con la participación del pueblo en las decisiones que éstos tomen. El análisis del discurso político tiene lugar en lo social y sólo se puede comprender en la interrelación entre la situación social, la acción, el actor y las estructuras sociales.

Se encontró convergencia en el grupo, en cuanto a que la política debe buscar el bienestar común de los ciudadanos, dentro de condiciones de equilibrio y equidad: coinciden en la legitimación que se le da a los representantes del poder político a través del voto. Algunos de los participantes relacionan la política con las leyes y normas que regulan el comportamiento de la sociedad y con la delegación del poder en los representantes políticos, a fin de que sean éstos los que organizan y dirijan los destinos de Colombia como Nación.

El grupo coincidió en deslegitimar el poder político otorgado a los representantes, en la medida en que éstos excluyen la participación de la ciudadanía en las decisiones del Estado; de esta manera, la política es percibida como distante de la ciudadanía. Confluyen en sentimientos de exclusión, debido a que perciben la política como ajena a su realidad y que ésta, en lugar de orientarse hacia el bien común, es guiada por los partidos políticos hacia intereses particulares. Para los participantes la política se manifiesta en diferentes contextos, pero principalmente en la familia, la universidad, las empresas y en lugares donde se pueda originar cambio social.

De todas formas, hay una perspectiva global de que el voto es prácticamente el principal mecanismo de participación del cual hacen uso. Los participantes relacionan el voto con los procesos electorales y con la democracia a nivel institucional, siempre en función de la elección de los representantes del pueblo en el gobierno. En cuanto a la participación política, la mayoría de los participantes reconocen su escasa participación en el cuestionamiento de algunas de las decisiones que toma el gobierno y que afectan el bienestar de los ciudadanos.

Resaltan la importancia de que los ciudadanos tengan la oportunidad de conocer e informarse sobre lo que sucede dentro del sistema político para proponer alternativas de solución a los problemas que afectan a la sociedad. Adicionalmente, algunos de los estudiantes manifiestan que "es necesario el uso del voto, como mecanismo de participación, sea hecho de una manera consciente y crítica para no votar por votar o por pura presión". De esta manera, según ellos, la participación política tendrá como fruto la transformación o cambio de la situación del país. No obstante, se requiere que se constituyan en sujetos activos, bien sea siendo miembros de algún grupo político que modifique los actos erróneos del Estado.

Con respecto a los procesos políticos en los que participan los jóvenes, los participantes del estudio manifestaron que tienen conocimiento sobre programas de organizaciones comunitarias, procesos electorales y en concejos juveniles. Resaltan la importancia de iniciar este conocimiento a través de los procesos de participación en las instituciones educativas como la elección de personeros. También resaltan la participación comunitaria como ediles, concejos comunales, en las juntas de acción comunal.

Pese a lo anterior, reconocen que hay un amplio desconocimiento de los mecanismos y de las instancias de participación de los jóvenes; atribuyen este aspecto a la desconfianza en la política y en los políticos, a la corrupción, a la inclusión intencionada de unos pocos jóvenes y a la exclusión de la mayoría. Algunos de los participantes conocen de la participación de jóvenes en movimientos juveniles o estudiantiles, en programas de la Presidencia de la República como Jóvenes en Acción; en los barrios y localidades a través de los consejos de juventud. Sin embargo, resaltan que es cada vez mayor la resistencia juvenil por participar en la política, ya que existe una apatía generalizada. 
Al reflexionar sobre el tema de la democracia, como un elemento importante en la constitución de lo político, los participantes consideran que está referida a la participación del pueblo en las decisiones del Estado. Esta participación está mediada por la igualdad de derechos, la equidad y la justicia. La democracia se relaciona con un consenso de ideas que beneficien a la sociedad; de igual forma, ésta implica libertad de elegir, igualdad y cumplimiento de derechos y deberes, equidad en las decisiones tomadas, elegir para ser gobernado. Por último hay un acuerdo en que la participación democrática debe apuntar al bienestar común.

Por supuesto, para algunos de los participantes, desde su mirada subjetiva sobre la política y el papel que la democracia juega en ella, la consideran como una máscara frente a la que los ciudadanos, supuestamente, comparten la libertad, pero siempre prevalecen los intereses de algunas minorías sobre los intereses de los ciudadanos. Asimismo, para la mayoría de los participantes, el único proceso democrático que conocen es la elección a través del voto; sienten que se vulnera el proceso de participación democrática mediante la corrupción previa y durante los procesos de elecciones públicas.

Desde su subjetividad reconocen la importancia de configurarse como sujetos políticos activos, de generar relaciones en las que puedan participar, conocer, aprender y, sobre todo, comprender las dinámicas contemporáneas de la política. La participación ampliada les permitiría discutir, expresar, dialogar y cuestionar las decisiones o temas de las agendas políticas.

La interacción política tiene para los participantes un carácter de enseñanza, en la medida en que reconocen que a través de la socialización los individuos aprenden sobre el sentido de la vida en sociedad y de su compromiso en ella, pues hasta ahora hay jóvenes y adultos que se ponen al margen de la política aduciendo simplemente que no les gusta o que no entienden nada de ella.

La configuración como sujetos políticos implica relacionarse con otras personas, aprender a convivir bajo una serie de normas, reglas y parámetros impuestos por la sociedad. La mayoría de los participantes coinciden en que la socialización permite la construcción y la estructuración de este proceso. Los participantes en el estudio resaltan que los medios de comunicación también ejercen influencia en la construcción de la subjetividad política, lo cual está unido a lo que se observa en la actuación de los políticos y la situación del país, se va estructurando un sentido particular de la política.

Los estudiantes piensan que la formación académica debe contribuir a la definición de la política y a la constitución de los jóvenes como sujetos pensadores y actuantes en la esfera de la política. También enfatizan en el papel que deben cumplir la familia y los medios de comunicación como agentes formadores, tanto de la subjetividad como de la objetividad política.

Al recuperar su experiencia política, algunos de los estudiantes consideran que el acercamiento es escaso y precario, debido a que no se han interesado en el tema. Según las respuestas de los participantes, las personas que han influido en el acercamiento al tema político y con las que generalmente hablan de política son los padres y familiares, compañeros de trabajo o universidad, amigos y algunos profesores.

Algunos enfatizan que su distanciamiento de la esfera de la política se debe a la constante corrupción que ven, al estado de crisis de credibilidad que ha tenido en los últimos años y a la inequidad social que propician las castas políticas dominantes en el país.

\section{Discusión}

Bruner (1997) plantea la narrativa como un eje de interpretación en la formación subjetiva de la realidad; mediante las narrativas se puede comprender la influencia del mundo sobre el sentido y significado personal. Las construcciones narrativas dan forma a la realidad que crean. La experiencia de cada sujeto sobre los asuntos humanos, como la política, toma la forma de las narraciones que se emplean para contar cosas sobre ella. 
El conocimiento personal, de grupo, cultural se constituye en fuente de contenido de las narrativas tanto individuales como colectivas. Las narrativas tienen lugar en lo social y sólo se pueden comprender en la interrelación entre la situación social, la acción, los actores, las estructuras sociales y la historia (Josselson \& Lieblich, 1993).

Dado lo anterior, el presente trabajo fue enfocado a identificar los procesos de subjetividad y participación política en estudiantes de Psicología en Bogotá. La subjetividad y la participación política se ubican dentro de los procesos de socialización (Berger \& Luckman, 1968/1999). En estos procesos de socialización se incluyen, además, la actitud política frente a la política y la democracia como elemento fundamental de la política.

La investigación arrojó unos resultados que contrastados con el marco teórico de referencia, permiten vislumbrar algunas ideas acerca de cómo el proceso de subjetividad influye en la participación política de los jóvenes participantes en el estudio (Berger \& Luckamn, 1999). Los resultados obtenidos a través la entrevista individual y de grupo focal muestran que la subjetividad política se constituye a partir de la experiencia y la percepción que cada uno de los participantes posee sobre la política con sus alcances y limitaciones, los políticos y sobre la forma en que la sociedad le presenta a los ciudadanos una realidad política que convoca a unos y aleja a otros (Calcagno \& Calcagno, 1999).

Según lo planteado por Berger \& Luckmann (1999), a partir de los otros significantes, que mediatizan los significados de la realidad, es que se construye la realidad subjetiva de cada individuo. A partir de la experiencia que posean otros en la política, los individuos construyen su sentido de lo político y a partir de esa construcción se puede garantizar o no la participación política de los jóvenes. Según los resultados obtenidos en esta investigación, el proceso de subjetividad política en los estudiantes se define por las percepciones, las creencias, las experiencias en su formación, tanto objetiva como subjetiva, de su condición de sujetos políticos. Resaltan el valor de las relaciones sociales como escenarios de discusión, argumentación e incluso de comprensión de las manifestaciones de la política en manos de los políticos y de los ciudadanos.

Por su parte, la socialización es parte fundamental para que el sujeto se haga partícipe en la política, de acuerdo con la manera en que se integre el individuo a la sociedad, a partir de la función mediatizadora de los agentes de socialización (Sabucedo, 1996). Los agentes de socialización u otros significativos son concebidos como los principales transmisores de la socialización política, tanto objetiva como subjetiva. Según lo anterior, se considera que el proceso de socialización constituye la base fundamental para que un individuo se haga o no partícipe de la política, entendiendo participación política como el conjunto de actividades, interacciones, comportamientos, acciones y actitudes que se dan en una sociedad en forma individual o colectiva, por parte de individuos, grupos, partidos e instituciones, las cuales van dirigidas a explicar, demandar, influir o a tomar parte en el proceso de decisiones políticas, en el reparto autoritario de valores (Sabucedo, 1996).

Según Benedicto y Morán (1993), la participación política no reviste mayor relevancia para los jóvenes, como resultado de su propia socialización, pero también de los límites del sistema de representación. Visto desde un ángulo negativo, los procesos anteriores involucran disolución de las identidades políticas colectivas en algunos jóvenes y la reducción de su participación como ciudadano y miembro de la sociedad. En otros casos, se evidencia una expansión de espacios culturales que los jóvenes apropian a través de sus discursos, de su acción y de su identidad para expresarse como sujetos políticos. Se puede decir que los jóvenes se distribuyen entre quienes no quieren saber nada de la política y entre quienes muestran interés a través de sus propios lenguajes, símbolos y formas de actuación en público.

Un aspecto de interés es el hecho de que en los resultados obtenidos se nota un solapamiento entre el concepto participación política y democracia, ya que pertenecer a un Estado democrático, el cual implica que el pueblo puede participar de las decisiones del Estado. Pese a 
esto, la vía de participación que resaltan con mayor frecuencia los estudiantes es el voto para la elección de los representantes políticos.

Adicionalmente, un factor de gran importancia y que influye en la decisión de los jóvenes de participar o no es la forma en que perciben la política. De acuerdo con los resultados y en relación con este aspecto, es común la referencia de los estudiantes sobre el problema de corrupción que embarga la política, lo que ha llevado al país a un estado de crisis, de falta de credibilidad y de ilegalidad, en cuanto a que se manejan intereses e influencias que no se hacen explícitas y que no apuntan a la igualdad y al bienestar de la ciudadanía.

Al respecto, la Alcaldía Mayor de Bogotá (2003) considera que los y las jóvenes han estructurado un pensamiento sobre la política que los distancia de ella por sus problemas, lo que la han llevado al desprestigio.

La participación ciudadana en procesos políticos, tanto a nivel local, como regional, nacional o supranacional, debe ser considerada como un elemento central en el funcionamiento de los sistemas democráticos. Como lo demuestran numerosos ejemplos históricos, la participación política de los ciudadanos es de suma importancia tanto para la manutención de la legitimidad del ordenamiento político del que se trate, como en relación con la capacidad de dichos sistemas para enfrentar y resolver problemas económicos, sociales y políticos (Bárcena, 1997).

A la hora de estudiar el tema de la participación política de los jóvenes, las tres cuestiones clásicas que se plantean son la del interés por la política, la de la confianza que depositan en instituciones vinculadas a ella y la de la participación activa en la política. Por otra parte, el interés por la política y la participación activa deben ser vistos tanto en su dimensión institucional -es decir, en la disposición de los ciudadanos a participar en formas convencionales e institucionalizadas en las actividad políticas- como las elecciones, la afiliación a partidos políticos, las candidaturas, la conformación de mesas o colegios electorales, así como en la dimensión extra-institucional, es decir, en formas no convencionales y no institucionalizadas de participación, por ejemplo, en forma de protestas, manifestaciones, peticiones, recolección de firmas, manifiestos, solicitadas en los periódicos o en las manifestaciones artísticas de resistencia a las formas de expresión de la política contemporánea.

\section{Referencias}

Alba, V. (1975). Historia social de la juventud. Barcelona: Plaza y Janes. S. A.

Arendt, H. (2001). ¿Qué es la política? Madrid: Paidós.

Balardini, S. (2000). La participación social y política de los jóvenes en el horizonte del nuevo siglo. Buenos Aires: CLACSO.

Bárcena, F. (1997). El oficio de la ciudadanía. Barcelona: Paidós.

Benedicto, J. Morán, M.L. (2003). Aprendiendo a ser ciudadanos. Madrid: Ministerio de trabajo y Asuntos sociales Injuve.

Berger, P. \& Luckmann, T. (1999). La construcción Social de la Realidad. Buenos Aires: Amorrortu.

Beuchot, M. (2002). Perfiles esenciales de la hermenéutica. Instituto de investigaciones filológicas. México: Universidad Nacional Autónoma de México.

Bonilla-Castro, E. \& Rodríguez, P. (1997). Más allá del dilema de los métodos. Colombia: Editorial Norma. Universidad de los Andes.

Brown, S. (1999). Psicología política desde la Subjetividad Naturalista. En M. Montero, J.M. Sabucedo, B. Sanders \& I. Ferreira. Psicología política del nuevo siglo. Sociedad Mexicana de Psicología Social. A. C.

Brewer, M. (2001). Political Psychology (V. 22. No. 1, 115-125). Ohio State: Ohio State University. Department of Psychology. 
Bruner, J. (1997). La educación puerta de la cultura. Madrid: Visor.

Bruner, J. (2002). La fábrica de historias. Buenos Aires: Fondo de Cultura Económica.

Calcagno, A. \& Calcagno, E. (1999). Para entender la política. Entre la ilusión de lo óptimo y la realidad de lo pésimo. Argentina: Catálogos.

Cohen, P. (1999). Rethinking the Youth Question. Hong Kong: Duke University Press.

D’Adamo, O., García, V. y Montero, M. (1995) (comps.). Psicología de la acción política. Barcelona: Paidós.

Denzin, N \& Lincoln, T. (2003). Strategies of Qualitative Inquire. Thousand Oaks: Sage Publications.

De Sousa, B. (1998). De la mano de Alicia. Bogotá: Siglo del Hombre Editores y Ediciones UNIANDES.

Ferrándiz, F. \& Feixa, C. (Eds.) (2005). Jóvenes sin tregua. Barcelona: Anthopos.

Garretón, M. (2000). Política y sociedad entre dos épocas. América Latina en el cambio de siglo. Rosario, Argentina: HomoSapiens.

Goolishian, H. \& Anderson, H. (1998). Narrativas y self. Algunos dilemas postmodernos de la psicoterapia. En Dora Fried Schnitman (Eds). Nuevos paradigmas, cultura y subjetividad. Madrid: Paidós.

Guattari, F. (1998). El nuevo paradigma estético. En Dora Fried Schnitman. Nuevos paradigmas, cultura y subjetividad. Madrid: Paidós.

Held, D. (1997). La democracia y el orden global. Madrid: Paidós.

Huergo, J. (2004). La formación del sujeto y los sentidos político-culturales de comunicación/ educación. En M.C. Laverde, G. Daza \& M. Zuleta. Debate sobre el sujeto. Perspectivas contemporáneas. Bogotá: Universidad Central (DIUC). Siglo del Hombre Editores.

Huntinngton, S. (1997). El orden político en las sociedades en cambio. Madrid: Paidós.

Josselson, R \& Lieblich, A. (1993). The Narrative Study of Lives. Newbury Park: Sage Publication.

Lechner, N. (1995). Los patios interiores de la democracia. Subjetividad y Política. México: Fondo de Cultura Económica.

Lechner, N. (Marzo de 1999). Desafío de un desarrollo humano: individualización y capital social. Foro Desarrollo y Cultura. Asamblea General del Banco Interamericano de Desarrollo (BID). París, pp. 1-27.

Lieblich, A., Tuval-Mashiach, R. \& Zilber, T. (1998). Narrative Research. California: Sage publications.

Levi, G. \& Schmitt, J.C. (1996). Historia de los jóvenes: de la antigüedad a la edad moderna (V. I y II). España: Ed. Taurus.

Maffesoli, M. (2004). La transfiguración de lo político. México: Herder.

Martín C., E. (1998). Producir la juventud. Madrid: Istmo.

Mead, G.H. (1982). Espíritu, persona y sociedad. Buenos Aires: Paidós.

Merlino, A. \& Roqué, G. (2004). Los nuevos jóvenes. Un estudio psicográfico de sus actitudes y estilos de vida. Argentina: Editorial Brujas.

Nateras D., A. (2002). Jóvenes, culturas e identidades urbanas. México: Universidad Autônoma Metropolitana- Miguel Angel Porrúa.

Pastor, J. (2002). Los movimientos antiglobalización neoliberal. En J.M. Robles (comp.). El reto de la participación. Movimientos sociales y organización. Madrid: Machado libros. 
Pereira, M. \& Villadiego, M. (2006). Entre miedos y goces. Bogotá: Editorial Pontificia Universidad Javeriana.

Pérez Islas, J. (2000). Visiones y versiones. Jóvenes, instituciones y políticas de juventud. En Jesús Martín-Barbero et al. Umbrales, cambios culturales, desafíos nacionales y juventud. Medellín: Corporación Región.

Pourtois, J.P. y Desmet, H. (1992). Epistemología $e$ instrumentación en Ciencias Humanas. Barcelona: Herder.

Rauber, I. (2006). Sujetos políticos. Colombia: Ediciones desde Abajo.
Restrepo, G., Ortiz, J.M., Parra, F.E. \& Medina, L.C. (1998). Saber y poder: socialización política y educativa en Colombia. Bogotá: ICFES.

Rozo, E. (1990). Evolución de las ideas políticas. Bogotá: Universidad Externado de Colombia.

Sabine, G.H. (2002). Historia de la teoría política. México: Fondo de Cultura Económica.

Sabucedo, J.M. (1996). Psicología Política. Madrid: Síntesis Psicológica.

Sartori, G. (1995). Teoría de la democracia. El debate contemporáneo. Madrid: Alianza Editorial.

Valles, M. (1997). Técnicas cualitativas de investigación social. Madrid: Síntesis Sociología. 